\title{
Interactions between Cytochrome c and DNA Strands Self-Assembled at Gold Electrode
}

\section{Ruojun Lao ${ }^{1,3}$, Lihua Wang ${ }^{1}$, Ying Wan ${ }^{1}$, Jiong Zhang ${ }^{1}$ Shiping Song ${ }^{1,}$, Zhizhou Zhang ${ }^{2, *}$, Chunhai Fan ${ }^{1}$ and Lin $\mathrm{He}^{3}$}

1 Shanghai Institute of Applied Physics, Chinese Academy of Sciences, Shanghai 201800, China E-mail: spsong@ sinap.ac.cn (for Shiping Song).

2 Tianjin University of Science and Technology, Tianjin 300040, China

E-mail: zhangzz@tust.edu.cn (for Zhizhou Zhang).

3 Bio-X Life Science Research Center, Shanghai Jiao Tong University, Shanghai 200030, China

* Authors to whom correspondence should be addressed. Tel: +86 215955 4610; Fax: +86 215955 6902

Received: 29 November 2006 / Accepted: 29 January 2007 / Published: 23 February 2007

\begin{abstract}
In this work, we reported the investigation on the interaction between DNA strands self-assembled at gold electrodes and an electron transfer protein, cytochrome c. We observed that cytochrome c exhibited well-defined electrochemistry in both double-stranded and single-stranded DNA films. This suggested that the electron transfer reaction of cytochrome c arose possibly due to the electron hopping along DNA strands rather than wiring along the double helix. We also compared the heterogeneous electron transfer rate of cytochrome $\mathrm{c}$ with that of a ruthenium complex, which further confirmed this mechanism.
\end{abstract}

Keywords: Cytochrome c; DNA; electrochemistry; gold electrodes; cyclic voltammetry

\section{Introduction}

Electron transfer (ET) of proteins plays a vital role in both photosynthesis that traps light energy and metabolism that oxidizes fuel. Virtually all living organisms can efficiently convert electrochemical energy to either a trans-membrane proton gradient or substrate consumption. This high efficiency of long-range ET has mostly been attributed to the well-controlled natural protein-protein interface. 
However, in contrast, ET of proteins at electrodes, i.e., artificial charged interfaces, is usually difficult.[1-4] Indeed, only in rare cases could one observe direct electron communication between proteins and bare electrodes. As a major step toward in-vitro protein ET, self-assembled monolayers (SAMs) that can functionalize solid surfaces in a highly controlled manner was introduced, which offered great opportunities to mimic natural protein-protein interfaces at electrode surfaces. Importantly, self-assembly of organosulfur compounds (alkanethiols) on gold electrode surfaces has led to successful electrochemical characterization of a variety of electron-transfer proteins in vitro.[5-7]

Despite this significant progress, there are still many fundamental issues that remain to be resolved. For example, electron coupling of protein-SAMs is still less efficient than that at the interface of natural protein partners. This arises possibly due to the existence of specific interactions between natural protein partners. For example, Millett and coworkers found that replacement of yeast cytochrome c (cyt c) with the protein of horse origin significantly attenuated ET between cyt c and yeast cytochrome c oxidase, which strongly implied the importance of naturally existing proteinprotein interactions.[8] Since catalytic activities of many enzymes are directly coupled with their ET reactivity,[9] enhancement of interfacial ET can, in principle, be translated into significant improvement in the performance of protein-based biosensors, or the efficiency of bioelectronic devices (e.g. biofuel cells).

In order to achieve highly efficient interfacial ET of proteins, Xiao et al. recently reported that glucose oxidase (GOD) could be electrically wired to a gold electrode, which was bridged by a conductive gold nanoparticle.[10] In this nano-wired system, they observed an ET turnover rate of $\sim 5000 \mathrm{~s}^{-1}$, which even exceeded the corresponding rate between GOD and its natural substrate, oxygen, by approximately an order of magnitude. In an alternative approach, Gray and coworkers employed a thiolated conjugated oligomer to wire the deeply buried, otherwise inaccessible, redox site of Arthrobacter globiformis amine oxidase (AGAO) to a gold electrode.[11] At this electrode surface self-assembled with $2.2 \mathrm{~nm}$-long molecular wires, AGAO exhibited reversible electrochemistry with an extremely high ET rate $\left(\mathrm{k}_{0}>4 \times 10^{4} \mathrm{~s}^{-1}\right)$. Analogous to conjugated oligomers, base-stacked oligonucleotides are possibly another medium for long-range ET.[12] Thiolated oligonucleotides have proven to spontaneously form well aligned monolayers at gold surfaces.[13, 14] According to the reports by Barton and coworkers, intercalative redox probes that were localized at a distance from the electrode surface could efficiently transfer electrons to and from the underlying electrode via $\pi-\pi$ interactions arising from base stacking within the double helix.[15] That's to say, the stacked aromatic heterocycles of the DNA duplex may serve as an efficient medium for coupling electron donors and acceptors over very long distances. More recently, they demonstrated that the electrochemistry of several iron-sulfur proteins and a flavoenzyme could also be mediated by double stranded DNA (dsDNA).[16] However, the certainty of DNA duplex's electron transfer ability is still in debate.

We are particularly interested in DNA-mediated protein ET, which should not only widen our view on interfacial protein ET but deepen our understanding of protein-DNA interactions. In this work, we report the electrochemical interrogation on the ET of cytochrome c (cyt. c), a well-studied model for protein electrochemistry, within self-assembled DNA films. Cyt. c plays an important role in the biological respiratory chain, whose function is to receive electrons from cytochrome $c$ reductase and deliver them to cytochrome $c$ oxidase. So the electrochemical study of cyt. c is very important. 
Whereas the voltammetric response of cyt. $c$ is quite poor at bare metal electrodes, most likely due to protein denaturation at the metal electrode surface leading to extremely slow electron-transfer kinetics. $[17,18]$ While previous studies suggested that direct electrochemistry of cyt. c was feasible at dsDNA-modified gold electrodes,[19] the exact ET mechanism remains ambiguous. Here, we found that, in contrast to the iron-sulfur proteins and flavoenzyme studied by Barton and coworker, cyt. c binds to DNA mainly via electrostatic interactions (not intercalation)[20] and its electrochemistry is electron hopping-based (not “wiring”[21, 22]).

\section{Results and discussion}

\subsection{Cyt c electrochemistry}
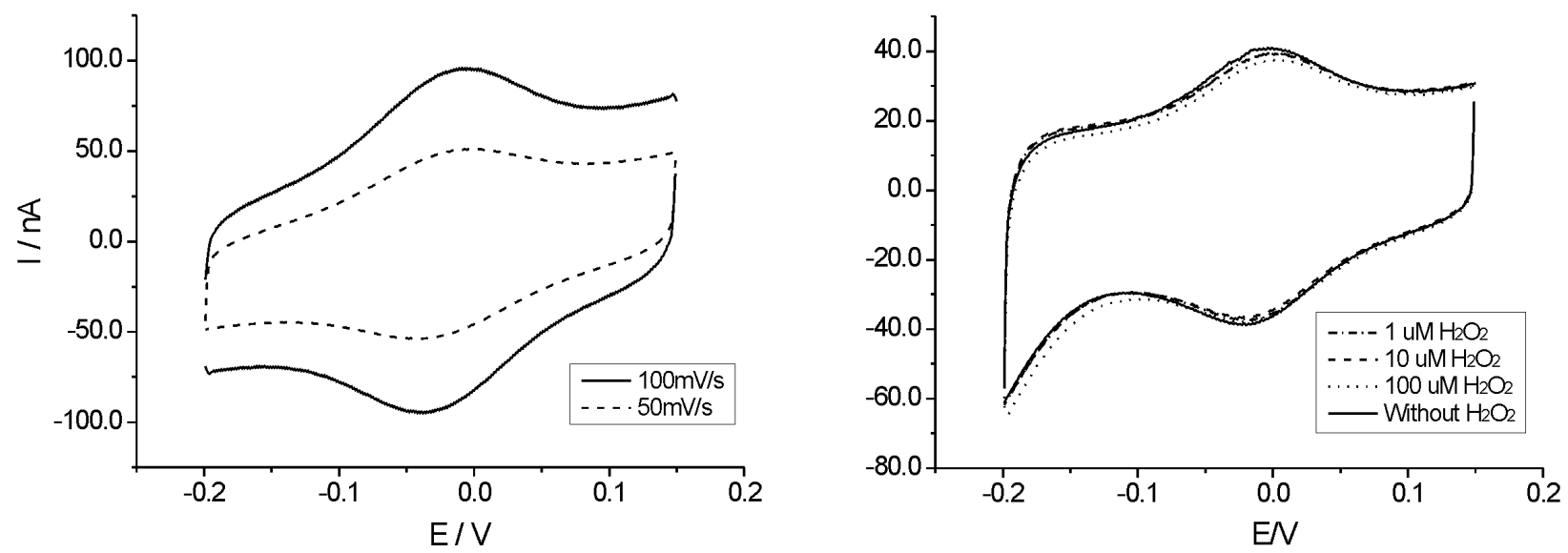

Figure 1. Cyclic voltammogram of cyt $\mathrm{c}$ adsorbed at DNA modified gold electrode $(\mathrm{A} ; 5 \mathrm{mM}$ Tris buffer, pH7.0, scan rate: $50 \mathrm{mV} / \mathrm{s}$ and $100 \mathrm{mV} / \mathrm{s}$ ) and cyt c/DNA modified electrodes (B; 5mM Tris$\mathrm{HCl}$, pH7.0 containing no $\mathrm{H}_{2} \mathrm{O}_{2}, 1 \mu \mathrm{M} \mathrm{H}_{2} \mathrm{O}_{2}, 10 \mu \mathrm{M} \mathrm{H}_{2} \mathrm{O}_{2}$ and $100 \mu \mathrm{M} \mathrm{H}_{2} \mathrm{O}_{2}$. Scan rate: $50 \mathrm{mV} / \mathrm{s}$ )

Thiolated ssDNA spontaneously form self-assembled monolayers on gold electrodes.[13, 14] Previous studies have demonstrated that these surface-confined DNA strands are predominantly in a well-aligned conformation that is nearly vertical to the electrode surface.[13, 14] Since DNA strands are highly negatively charged, multivalent cations can be electrostatically trapped within the DNA film. As is well known, cyt $\mathrm{c}$ is a positively charged protein at neutral $\mathrm{pH}$, arising due to several exposed protonable lysine at its surface. We thus incubated a DNA-modified electrode in a cyt c solution (30 $\mu \mathrm{M})$ with low ionic strength. Of note, high salt concentrations may screen electrostatic attraction between cyt $\mathrm{c}$ and DNA and minimize their interactions. After incubation of 20 min under repetitive potential scan, we obtained an electrode stably decorated with cyt c/DNA hybrid film. Significantly, cyt c could facilely communicate electrons with the gold electrode in this state, as manifested by the appearance of a pair of well-defined peaks in the CV experiment (Figure 1A). Note that the cyt c-DNA interaction is very strong since cyt $\mathrm{c}$ remains trapped in the DNA film even in a protein-free buffer solution and after being potential scanned for hours. The voltammetric response in E-buffer showed the stable redox transformation of the immobilized protein and the peak separation at this scan rate was about $20 \mathrm{mV}$, suggesting a fairly fast electron transfer reaction. The adsorption and redox 
transformation of cyt $\mathrm{c}$ were mostly attributed to the modification of SH-DNA. The negatively charged DNA strands effectively attracted positively charged cyt c via electrostatic interactions. In contrast, the gold electrode with MCH only exhibited weak adsorption and poor electrochemical behavior of cyt $\mathrm{c}$.

\subsection{Cyt c keeps its native structure at DNA modified electrodes}

It has been reported that proteins containing heme groups, such as HRP, hemoglobin and myoglobin, can catalyze the reduction of $\mathrm{H}_{2} \mathrm{O}_{2}[23,24]$ while cyt c cannot except when its native structure is either denatured or partial disturbed.[25, 26] As a result, it has been suggested that the catalytic ability for $\mathrm{H}_{2} \mathrm{O}_{2}$ can serve as a highly sensitive probe for the cyt c structure.[26, 27] In order to confirm that cyt c keeps its native structure at DNA modified electrode, we evaluated the catalytic effect on $\mathrm{H}_{2} \mathrm{O}_{2}$ by using cyt c absorbed at DNA modified electrodes. Interestingly, we observed the voltammograms of cyt c/DNA modified electrodes remained unchanged along with the elevation of $\mathrm{H}_{2} \mathrm{O}_{2}$ concentration, even at a high concentration of $100 \mu \mathrm{M}$ (Figure 1B). This clearly suggested that cyt c kept its native structure within DNA films, and that this system was appropriate for investigation of the electron transfer of cyt c.

\subsection{Dependence on DNA surface density}

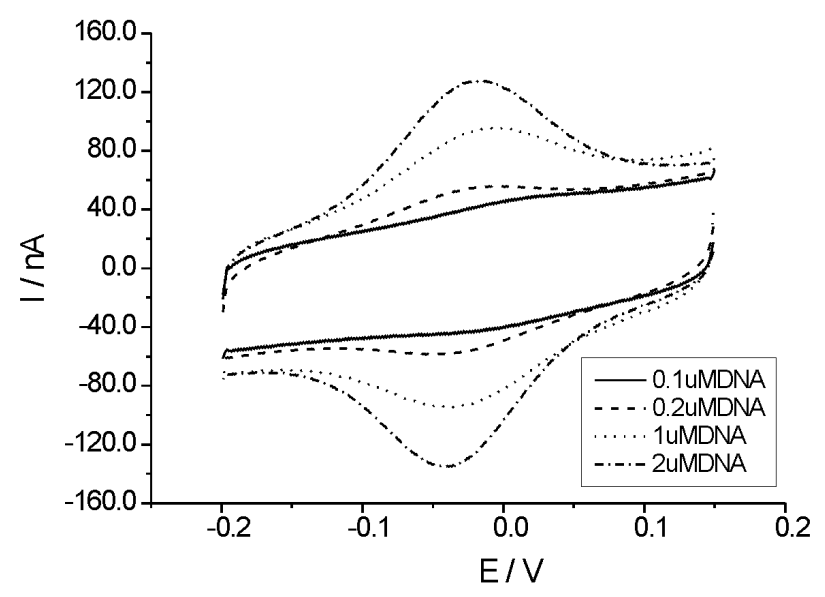

Figure 2. Cyclic votammograms of DNA modified electrode with different concentrations of DNA in immobilization buffers $(0.1,0.2,1,2 \mu \mathrm{M}) .5 \mathrm{mM}$ Tris buffer, $\mathrm{pH} 7.0$, scan rate: $100 \mathrm{mV} / \mathrm{s}$.

In order to unveil the role of self-assembly of DNA in adsorption process of cyt c, we turn to the relationship between the DNA surface density and the amount of electro-active cyt $\mathrm{c}$ absorbed on electrode. To obtain electrodes with different DNA surface density, a series of DNA I-buffers in which the concentrations of SH-DNA were $0.1 \mu \mathrm{M}, 0.2 \mu \mathrm{M}, 1 \mu \mathrm{M}$ and $2 \mu \mathrm{M}$ respectively, were prepared, while other conditions such as immobilization time, $\mathrm{MCH}$ treatment time and cyt $\mathrm{c}$ adsorption process remain unchanged. Note that higher concentration of SH-DNA in I-buffer resulted in increased number of thiolated oligonucleotides on electrodes. As shown in Figure 2, the denser SH-DNA was on surface, the larger the voltammetric response of cyt c. Both anodic and cathodic peak currents increased with 
the concentration of SH-DNA in I-buffer, demonstrating that the amount of electro-active protein was dependent on the surface density of SH-DNA.

\subsection{Electron transfer of cyt c at electrodes}

Figure 3 showed the CVs of cyt c/SH-DNA modified electrode at various scan rate of 0.05-10 V. The potentials and peak currents were dependent on the scan rate. The peak current linearly increased with the scan rate, suggesting that the reaction was a surface-controlled process. On the other hand, the peak-to-peak separation increased with the increase of scan rate. Marcus density-of-states (DOS) theory has been successfully applied to heterogeneous electron transfer reactions between metal electrodes and redox molecules attached on electrodes [28]. Based on this theory, heterogeneous electron transfer rate constants $\left(k_{\mathrm{et}}\right)$ can be estimated from the plot of peak separation versus the logarithm of scan rate. We thus obtained a $k_{\text {et }}$ of approximately 50 90 s ${ }^{-1}$. It is important to note that the $k_{\text {et }}$ is almost independent of the density of SH-DNA on electrodes (within experimental error).
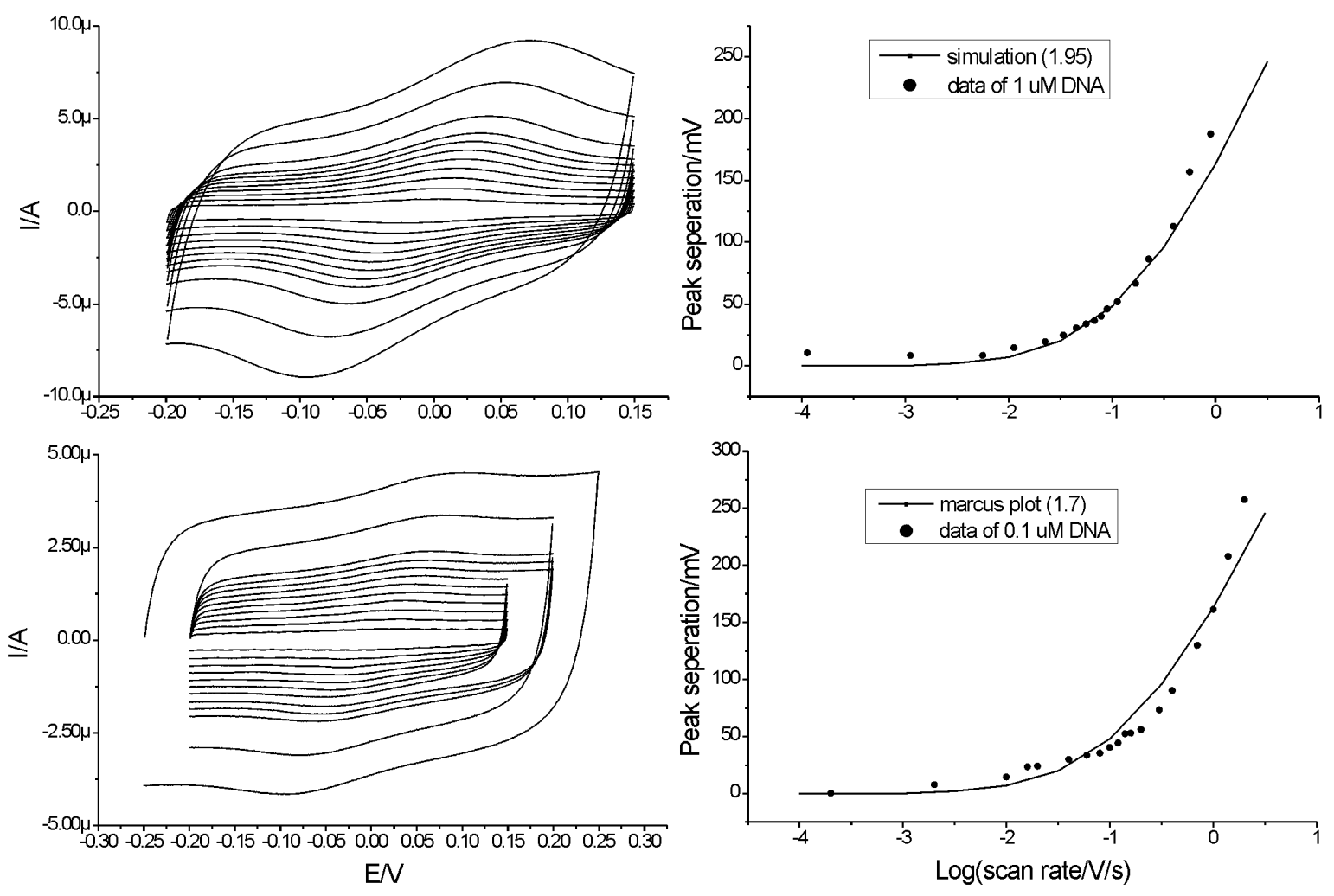

Figure 3. Cyclic voltammograms of cyt $\mathrm{c}$ at various scan rates ranging from 1-20 V/s (left) and simulation of Marcus plots (right). The upper and lower panels are for high (1 $\mu$ M SH-DNA) and low density (0.1 $\mu \mathrm{M}$ SH-DNA) DNA monolayers, respectively.

In order to evaluate the effect of DNA monolayer on the electrochemistry of cyt $\mathrm{c}$, we further prepared a dsDNA surface by immobilizing hybridized dsDNA on gold electrodes. We observed similar electrochemical reactions of cyt c. As shown in Figure 4, the heterogeneous electron transfer 
rate of cyt $\mathrm{c}$ at dsDNA modified electrodes was at the same order as that at ssDNA modified electrodes. This situation is contrast to those observed by Barton and coworkers. They noticed that intercalative dyes (e.g. methylene blue) underwent facile electron transfer reactions when intercalated in double stranded DNA, however even a single mismatch in base pairing significantly attenuated the electron transfer.[15] The fact that we observed well-defined electrochemistry for cyt $\mathrm{c}$ even with ssDNA strongly suggested that double helix was not important for transferring electrons of cyt c.
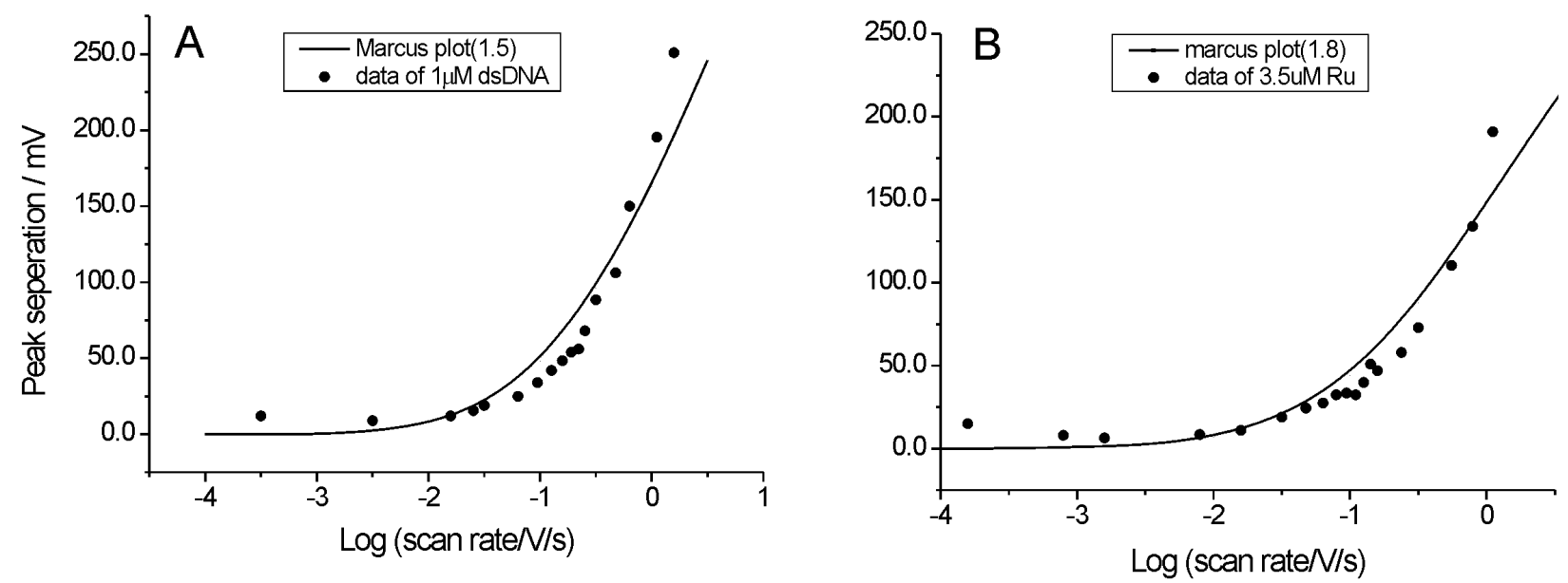

Figure 4. Data from CVs of cyt c (A) and RuHex (B) on dsDNA monolayer and its simulation to Marcus plots.

As a step further, we also compared the electron transfer rate of cyt c with that of RuHex. Previous studies have demonstrated that RuHex-DNA binding was completely based on electrostatic interactions, because it lacks planar organic groups that can intercalatively insert into DNA base pairs. As a result, electron transfer reaction of RuHex bound to DNA monolayers is solely due to the hopping along DNA strands, instead of any wiring effect.[29] We observed that the rate of RuHex redox $\left(k_{\text {et }}\right.$ $=60 \mathrm{~s}^{-1}$ ) is similar to that of cyt $\mathrm{c}$ (Figure 4), which further confirm that electron transfer of cyt $\mathrm{c}$ at the DNA modified electrodes is mostly associated with the hopping rather than wiring along DNA strands. It is probably due to the binding mechanism between DNA and cyt. c, which is more likely to be electrostatic interactions rather than intercalation in experiments of Barton et al. Although they also demonstrate the dsDNA mediated electrochemistry of several iron-sulfur proteins[16], the salt concentration of the testing buffer they employ is much higher, to the extent that can prevent electrostatic interactions between proteins and DNA[30]. Therefore, there must be some other binding patterns between dsDNA and these proteins.

\section{Experimental}

\subsection{Materials}

DNA oligonucleotides were purchased from Sangon Inc. (Shanghai). The sequences are 5'-SHCACGA CGTTG TAAAA CGACG GCCAG-3' (thiolated probe at 5' terminus with a C6 spacer, SHssDNA); and 5'-CTGGC CGTCG TTTTA CAACG TCGTG-3' (complementary-DNA). Tris(hydroxymethyl)-aminomethane was purchased from Cxbio biotechnology Ltd. 6-Mercapro-1- 
hexanol (MCH), cytochrome c from horse heart, Hexaammineruthenium (III) chloride $\left(\left[\mathrm{Ru}\left(\mathrm{NH}_{3}\right)_{6}\right]^{3+}\right.$, RuHex) and tris(2-carboxy-ethyl) phosphine hydrochloride (TCEP) were from Sigma. All solutions were prepared with nanopure water $(18 \mathrm{M} \Omega \bullet \mathrm{cm}$ resistivity) from a Millipore MilliQ system.

The buffers involved in this work are as follows. DNA immobilization buffer (I-buffer): $10 \mathrm{mM}$ TrisHCl, $1 \mathrm{mM}$ EDTA, $1.0 \mathrm{M} \mathrm{NaCl}$ and $1 \mathrm{mM}$ TCEP (pH 8.0). Note that TCEP was employed to cleave disulfides. DNA hybridization buffer (H-buffer): $10 \mathrm{mM}$ trisHCl, $1 \mathrm{mM}$ EDTA and $1.0 \mathrm{M} \mathrm{NaCl}$ (pH 8.0). Buffer for electrochemistry (E-buffer): 5mM Tris-HCl buffer, $\mathrm{pH}$ 7.0.

\subsection{Pretreatment of electrodes and DNA immobilization.}

Gold electrodes (2 $\mathrm{mm}$ in diameter, $\mathrm{CH}$ Instruments Inc.) were first polished on microcloth (Buehler) with Gamma micropolish deagglomerated alumina suspension $(0.05 \mu \mathrm{m})$ for $5 \mathrm{~min}$. These electrodes were then sonicated in ethanol and Milli-Q water for $5 \mathrm{~min}$, respectively. Finally, the electrodes were then electrochemically cleaned to remove any remaining impurities.[31-33] After drying with nitrogen, electrodes were subjected to oligonucleotides of appropriate concentrations for 14-16 hours, followed by a 2-hour deposition in $1 \mathrm{mM} \mathrm{MCH}$ in water.[34] DNA duplex was prepared by hybridization of the two complementary sequences $(0.5 \mu \mathrm{M}$ each) in H-buffer for at least 1 hour, and then be ready for immobilization at surfaces.

\subsection{Electrochemical measurements}

Cyclic voltammetry $(\mathrm{CV})$ and Alternating Current voltammetry (ACV) were performed on a $\mathrm{CH}$ Instruments model 430 eletrochemical analyzer. A three-electrode cell consisting of $\mathrm{Ag} / \mathrm{AgCl}$ reference electrode and platinum counter electrode was used for all electrochemical measurements. For cyt. c adsorption a $10 \mathrm{mM}$ Tris buffer $\mathrm{pH} 7.0$ was used. The protein concentration was $30 \mu \mathrm{M}$ and the electrode was cycled for about $20 \mathrm{~min}$ in the potential range from $-250 \mathrm{mV}$ to $+250 \mathrm{mV}$ (vs. $\mathrm{Ag} / \mathrm{AgCl}$ ). Then the electrode was transferred to the protein free E-buffer to take the measurement. The E-buffers were thoroughly purged with pure nitrogen before experiments.

\section{Conclusion}

In summary, we studied the electron transfer properties of cyt c within self-assembled DNA films. We found that the electron transfer of cyt $\mathrm{c}$ within ssDNA films was as efficient as that within dsDNA films. In addition, electron transfer of RuHex, a simple cation that cannot intercalate into dsDNA, also showed comparable electron transfer kinetics. We thus conclude that electron transfer of cyt $\mathrm{c}$ is electron hopping based, rather than a "wiring" process along DNA double helix.

\section{Acknowledgments}

We thank the financial support from National Natural Science Foundation (60537030, 20404016 and 30570401), Ministry of Science and Technology (2006CB933000), Shanghai Municipal Commission for Science and Technology (0652nm006, 0652nm016 and 06ZR14106), Shanghai Rising-Star Program and Chinese Academy of Sciences. 


\section{References}

1. Ryan, M. D.; Bowden, E. F. and Chambers, J. Q. Dynamic electrochemistry: methodology and application. Anal. Chem., 1994, 66, 360R-427R.

2. Hirst, J. and Armstrong, F. A. Fast-scan cyclic voltammetry of protein films on pyrolytic graphite edge electrodes: characteristics of electron exchange. Anal. Chem., 1998, 7, 5062-5071.

3. Stellwagen, E. Haem exposure as the determinate of oxidation-reduction potential of haem proteins. Nature, 1978, 275, 73-74.

4. Voet, D. and Voet, J. G. Biochemistry 2nd Edition. John Wiley\&Sons,Inc., New York, 1993.

5. Baymann, F.; Moss, D. A. and Mantele, W: An electrochemical assay for the characterization of redox proteins from biological electron transfer chains. Anal. Biochem., 1991, 199, 269-274.

6. Armstrong, F. A.; Camba, R.; Heering, H. A. et al.: Fast voltammetric studies of the kinetics and energetics of coupled electron-transfer reactions in proteins. Faraday Discuss., 2000, 191-203; discussion 257-168.

7. Elliott, S. J.; McElhaney, A. E.; Feng, C.; Enemark, J. H. and Armstrong, F. A. A voltammetric study of interdomain electron transfer within sulfite oxidase. J. Am. Chem. Soc., 2002, 124, 1161211613.

8. Hahm, S.; Durham, B. and Millett, F. Photoinduced electron transfer between cytochrome c peroxidase and horse cytochrome $c$ labeled at specific lysines with (dicarboxybipyridine)(bisbipyridine)ruthenium(II). Biochemistry, 1992, 31, 3472-3477.

9. Mure, M.; Mills, S. A. and Klinman, J. P. Biochemistry, 2002, 41, 9269-9278.

10. Huang, Y.; Zhang, W.; Xiao, H and Li, G. An electrochemical investigation of glucose oxidase at a CdS nanoparticles modified electrode. Biosens. Bioelectron., 2005, 21, 817-821.

11. Contakes, S. M.; Juda, G. A.; Langley, D. B. et al.: Reversible inhibition of copper amine oxidase activity by channel-blocking ruthenium(II) and rhenium(I) molecular wires. Proc. Natl. Acad. Sci. U. S. A., 2005, 102, 13451-13456.

12. Chen, X.; Ruan, C.; Kong, J. and Deng, J. Spectroelectrochemical investigation of direct electron transfer between resting horseradish peroxidase and its oxidation states promoted by DNA. Fresenius J Anal. Chem., 2000, 367, 172-177.

13. Steel, A. B.; Herne, T. M. and Tarlov, M.J. Electrochemical quantitation of DNA immobilized on gold. Anal. Chem., 1998, 70, 4670-4677.

14. Thiel, A. J.; Frutos, A. G.; Jordan, C.E.; Corn, R.M. and Smith, L.M. In Situ Surface Plasmon Resonance Imaging Detection of DNA Hybridization to Oligonucleotide Arrays on Gold Surfaces. Anal. Chem., 1997, 69, 4948-4956.

15. Kelley, S. O.; Boon, E. M.; Barton, J. K.; Jackson, N. M. and Hill, M. G. Single-base mismatch detection based on charge transduction through DNA. Nucleic. Acids Res., 1999, 27, 4830-4837.

16. Boal, A. K.; Yavin, E.; Lukianova, O. A.; O'Shea, V. L.; David, S. S. and Barton, J. K. DNAbound redox activity of DNA repair glycosylases containing [4Fe-4S] clusters. Biochemistry, 2005, 44, 8397-8407.

17. Reed, D. E. and Hawkridge, F. M. Direct electron transfer reactions of cytochrome c at silver electrodes. Anal. Chem., 1987, 59, 2334-2339.

18. Hawkridge, F. M. and Taniguchi, I. Comments Inorg. Chem., 1990, 17, 163-187. 
19. Lisdat, F.B.; Ge, B.K.; Ehrlich, A.H.; Bienert, H. and Scheller, F.W. Nucleic Acid-Promoted Electron Transfer to Cytochrome c. Electroanalysis, 2001, 13, 1225-1230.

20. Uosaki, K.; Kondo, T.; Okamura, M. and Song, W.: Electron and ion transfer through multilayers of gold nanoclusters covered by self-assembled monolayers of alkylthiols with various functional groups. Faraday Discuss., 2002, 373-389; discussion 441-362.

21. Creager, S.; Yu, C. J.; Bamdad, C. et al.: Electron Transfer at Electrodes through Conjugated “Molecular Wire”Bridges. J. Am. Chem. Soc., 1999, 121, 1059-1064.

22. Samanta, M. P.; Tian, W.; Datta, S; Henderson, J. I. and Kubiak, C. P. Electronic conduction through organic molecules. Physical Review. B. Condensed Matter., 1996, 53, R7626-R7629.

23. D'Agnillo, F.; Wood, F.; Porras, C.; Macdonald, V. W. and Alayash, A. I. Effects of hypoxia and glutathione depletion on hemoglobin- and myoglobin-mediated oxidative stress toward endothelium. Biochim. Biophys. Acta, 2000, 1495, 150-159.

24. Huang, H.; Hu, N.; Zeng, Y. and Zhou, G. Electrochemistry and electrocatalysis with heme proteins in chitosan biopolymer films. Anal. Biochem., 2002, 308, 141-151.

25. Zhao, G. C.; Yin, Z. Z. and Wei, X. W. Direct electrochemistry of cytochrome c on a multi-walled carbon nanotubes modified electrode and its electrocatalytic activity for the reduction of $\mathrm{H}_{2} \mathrm{O}_{2}$. Electrochemistry Com., 2005, 7, 256-260.

26. Diederix, R. E. M.; Ubbink, M. and Canters, G. W.: Peroxidase Activity as a Tool for Studying the Folding of c-Type Cytochromes. Biochemistry, 2002, 41, 13067-13077.

27. Diederix, R. E. M.; Ubbink, M. and Canters, G. W. Effect of the Protein Matrix of Cytochrome c in Suppressing the Inherent Peroxidase Activity of Its Heme Prosthetic Group. Chembiochem, 2002, $1,110-112$.

28. Finklea. H. O.; Yoon, K.; Chamberlain, E.; Allen, J. and Haddox, R. Effect of the Metal on Electron Transfer across Self-Assembled Monolayers. J. Phy. Chem. B, 2001, 105, 3088-3092.

29. Lao, R.; Song, S.; Wu, H. et al.: Electrochemical Interrogation of DNA Monolayers on Gold Surfaces. Anal. Chem., 2005, 77, 6475.

30. Hamilton, R. W. and Lloyd, R. S. Modulation of the DNA scanning activity of the Micrococcus luteus UV endonuclease. J. Bio. Chem., 1989, 264, 17422-17427.

31. Fan, C.; Zhong, J.; Guan, R. and Li, G. Direct electrochemical characterization of Vitreoscilla sp. Haemoglobin entrapped in organic films. Biochim. Biophys. Acta-Proteins Proteom., 2003, 1649, 123-126.

32. Hoogvliet, J. C.; Dijksma, M.; Kamp, B. and Bennekom, van W. P. Anal. Chem., 2000, 72, 20162021

33. Janek, R. P.; Fawcett, W. R. and Ulman, A. J. Phys. Chem. B, 1997, 101, 8550-8558.

34. Fan, C.; Plaxco, K. W. and Heeger, A. J. Electrochemical interrogation of conformational changes as a reagentless method for the sequence-specific detection of picomolar DNA. Proc. Natl. Acad. Sci., U.S.A., 2003, 100, 9134-9137.

(C) 2007 by MDPI (http://www.mdpi.org). Reproduction is permitted for noncommercial purposes. 